\title{
Mixed Giardia duodenalis assemblage A and E infections in calves
}

\author{
T. Geurden ${ }^{\mathrm{a}, *}$, P. Geldhof ${ }^{\mathrm{a}}$, B. Levecke ${ }^{\mathrm{a}}$, C. Martens ${ }^{\mathrm{b}}$, D. Berkvens ${ }^{\mathrm{c}}$, \\ S. Casaert ${ }^{\text {a }}$, J. Vercruysse ${ }^{\text {a }}$, E. Claerebout ${ }^{\text {a }}$ \\ ${ }^{a}$ Laboratory of Parasitology, Faculty of Veterinary Medicine, Ghent University, Salisburylaan 133, B-9820 Merelbeke, Belgium \\ ${ }^{\mathrm{b}}$ Bioinformatics and Evolutionary Genomics, Department of Molecular Genetics, Ghent University, Technologiepark 927, B-9052 Ghent, Belgium \\ ${ }^{c}$ Department of Veterinary Epidemiology, Prince Leopold Institute of Tropical Medicine, Nationalestraat 155, B-2000 Antwerp, Belgium
}

Received 12 June 2007; received in revised form 13 July 2007; accepted 17 July 2007

\begin{abstract}
A molecular epidemiological study was conducted on 100 dairy (499 calves) and 50 beef (333 calves) farms in Belgium to estimate the prevalence of different Giardia duodenalis assemblages in calves younger than 10 weeks of age. Positive samples from the epidemiological study and from a previous clinical study were selected and genotyped based on the amplification of the $\beta$-giardin gene. To investigate the occurence of mixed assemblage A and E infections in calves, a novel assemblage-specific PCR was developed based on the triose-phosphate isomerase gene. The prevalence was 22\% (95\% Probability Interval (PI): $12-34 \%$ ) in dairy calves and $45 \%$ (95\% PI: $30-64 \%$ ) in beef calves. In total, 120 Giardia-positive samples from dairy and beef calves collected in the epidemiological study and from clinically affected calves were identified based on the amplification of the $\beta$-giardin gene. Overall G. duodenalis assemblage E was more prevalent (in 64\% of the samples), although the majority $(59 \%)$ of the dairy calves were infected with $G$. duodenalis assemblage A. Furthermore, mixed $G$. duodenalis assemblage $\mathrm{A}$ and $\mathrm{E}$ infections were identified in $31 \%$ of the calf samples $(n=101)$ using the assemblage-specific PCR. We believe this is the first report of mixed infections in calves, and the results of the present study indicate that calves, although mainly infected with the host-specific $G$. duodenalis assemblage E, are frequently infected with the zoonotic assemblage A, either as a mixed or mono-infection, suggesting that calves might be underestimated as a potential zoonotic reservoir for human infections.

(c) 2007 Australian Society for Parasitology Inc. Published by Elsevier Ltd. All rights reserved.
\end{abstract}

Keywords: Giardia; Mixed infections; Cattle; Molecular epidemiology; Belgium

\section{Introduction}

Worldwide, the prevalence of Giardia in calves younger than 6 months of age varies between 20\% (Wade et al., 2000 ) and $73 \%$ (Olson et al., 1997). Studies in the United States, Canada, Australia and Japan suggest that the public health risk from cattle is minimal (Caccio et al., 2005), although the zoonotic Giardia duodenalis assemblage A has been reported in up to $20 \%$ of either pre-weaned calves (O'Handley et al., 2000; Appelbee et al., 2003; Becher et al., 2004; Trout et al., 2004; Itagaki et al., 2005), post-weaned calves (Trout et al., 2005) or adult cattle (Trout et al., 2006;

\footnotetext{
* Corresponding author. Tel.: +32926473 93; fax: +3292647496.

E-mail address: thomas.geurden@ugent.be (T. Geurden).
}

Uehlinger et al., 2006). Occasionally, G. duodenalis assemblage B has been reported in calves (van Keulen et al., 2002; Lalle et al., 2005). In Europe, only a few molecular epidemiological studies have been conducted on calves. In Denmark, the majority of the isolates from cattle were identified as $G$. duodenalis assemblage E (Langkjaer et al., 2007). In contrast, a high prevalence of the zoonotic $G$. duodenalis assemblage $\mathrm{A}$ infections was reported in cattle in Italy (Berrilli et al., 2004; Lalle et al., 2005). Given the intensive nature of the cattle industry and the high population density in Europe, anthropozoonotic transmission is likely to occur frequently. Moreover, the occurrence of mixed G. duodenalis assemblage $\mathrm{A}$ and $\mathrm{E}$ infections in calves has never been considered. Since the exponential nature of PCR favours the amplification of the most abundant species present in a sample and due to the high fre- 
quency of transmission in calves, the host-specific G. duodenalis assemblage $\mathrm{E}$ is likely to out-compete underlying $G$. duodenalis assemblage A infections, as has been described in dogs (Hopkins et al., 1997).

Therefore, a molecular epidemiological study was conducted in Belgium to estimate the prevalence of Giardia in dairy and beef calves, with emphasis on the occurrence of mixed $G$. duodenalis assemblage A and $\mathrm{E}$ infections, using a novel assemblage-specific PCR based on the triose-phosphate isomerase (tpi) gene. The results of the assemblage-specific PCR were compared with the results of a previously described PCR for the amplification of the $\beta$-giardin gene (Lalle et al., 2005). In addition to the dairy and beef samples from the epidemiological study, samples from symptomatic calves from a previous clinical study (Geurden et al., 2006) were included to study a possible assemblage-specific pathogenicity.

\section{Materials and methods}

\subsection{Study design}

In a cross-sectional epidemiological study, 100 randomly selected dairy farms and 50 beef farms in the province of East-Flanders, Belgium, were visited on a single occasion between September 2001 and August 2005. All dairy calves were Holsteins or local breeds mixed with Holsteins. All beef calves were Belgian blue and white (BBW) or local breeds mixed with BBW. Faecal specimens were collected from all calves aged from newborn to 10 weeks of age present on the farm at the time of the visit and transported to the laboratory. A commercially available direct immunofluorescence assay (IFA; Merifluor Cryptosporidium/Giardia kit; Meridian Diagnostics Inc., Cincinnati, OH, USA) was used to detect Giardia cysts, as previously described (Geurden et al., 2004).

\subsection{Molecular characterisation}

Giardia-positive isolates from dairy and beef calves from the epidemiological study were selected for molecular typing. Furthermore, 27 samples from calves with clinical giardiosis, not included in the epidemiological study and younger than 1 year, were selected (Geurden et al., 2006). DNA was extracted using the QIAamp Stool Mini Kit (Qiagen) according to the manufacturer's instructions, incorporating an initial step of three freeze-thaw cycles (freezing in liquid nitrogen for $5 \mathrm{~min}$ and heating at $95^{\circ} \mathrm{C}$ for $5 \mathrm{~min}$ ) in the protocol to maximise cyst lysing. The eluted DNA was dissolved in $15 \mu$ l of ultra-pure water.

For the amplification of the Giardia $\beta$-giardin gene, the PCR protocol was performed as previously described (Lalle et al., 2005). Amplification products were subsequently visualized on $1.5 \%$ agarose gels with ethidium bromide. A positive (Plasmid DNA) and negative (PCR water) control sample was included in each sequence of PCRs. PCR products were purified using the Qiaquick purification kit
(Qiagen) and fully sequenced using the Big Dye Terminator V3.1 Cycle sequencing Kit (Applied Biosystems). Sequencing reactions were analysed on a 3100 Genetic Analyzer (Applied Biosystems) and assembled with Seqman II (DNASTAR, Madison, WI, USA). Sequences were compared with known sequences by BLAST-analysis (Altschul et al., 1997) against the NCBI database (http:// www.ncbi.nlm.nih.gov/BLAST). Furthermore, phylogenetic analysis was performed on more conserved positions of the nucleotide alignment created with MUSCLE (Edgar, 2004). The sequence alignments were manually improved using BioEdit (Hall, 1999). Distance matrices were calculated based on the Jukes and Cantor correction and trees were constructed with the neighbour-joining algorithm using the software package TREECON (Van de Peer and De Wachter, 1994). Bootstrap analysis with 500 replicates was performed to test the significance of the nodes. The sequence alignments were used to designate the assemblage to the respective sample.

For the assemblage-specific amplification of the Giardia tpi gene the primary PCR was performed as described by Sulaiman et al. (2003). In the nested PCR, new assemblage-specific primers were employed, using a 1/10 dilution of the first PCR as template. G. duodenalis assemblage Aspecific primers (Af: $5^{\prime}-$ CGC CGT ACA CCT GTC A-3' and Ar: 5'-AGC AAT GAC AAC CTC CTT CC-3') were designed based on Genbank Accession Nos. AY368157 to AY368161, GIU57897 and AY655704, to amplify a $332 \mathrm{bp}$ PCR product. Furthermore, G. duodenalis assemblage Especific primers (Ef: $5^{\prime}$-CCC CTT CTG CCG TAC ATT TAT-3' and Er: 5'-GGC TCG TAA GCA ATA ACG ACT $\mathrm{T}^{-3^{\prime}}$ ) were designed based on Genbank Accession Nos. AY228645 to AY228647, and AY655705 to AY655706, for the amplification a 388 bp PCR product. The reaction mixture for the secondary species-specific tpi PCR consisted of a master mix containing $1.25 \mathrm{U}$ of Taq DNA polymerase in $2.5 \mu \mathrm{l}$ standard Taq buffer (GE Healthcare, Buckinghamshire, UK), $0.5 \mu 1$ deoxyribonucleotide triphosphate (dNTP) mixture, BSA to a final concentration of $0.1 \mu \mathrm{g} \mathrm{BSA} / \mu 1$ reaction mixture, $3 \mathrm{mM} \mathrm{MgCl}_{2}$, $0.2 \mu \mathrm{M}$ of each primer and $2.5 \mu \mathrm{l}$ of template DNA in a total volume of $25 \mu$ l. Subsequent steps were initial denaturation for $10 \mathrm{~min}$ at $94^{\circ} \mathrm{C}$, followed by 35 cycles of denaturation for $45 \mathrm{~s}$ at $94{ }^{\circ} \mathrm{C}$, annealing for $45 \mathrm{~s}$ at $64{ }^{\circ} \mathrm{C}$ for the assemblage A-specific primers or $67^{\circ} \mathrm{C}$ for the assemblage E-specific primers and an extension for $45 \mathrm{~s}$ at $72{ }^{\circ} \mathrm{C}$. Amplification products were subsequently visualised on $1.5 \%$ agarose gels with ethidium bromide. A positive (Plasmid DNA) and negative (PCR water) control sample were included in each sequence of PCRs. To verify the specificity of the tpi primers, DNA from $G$. duodenalis assemblages A, B and E (kindly provided by Simone Caccio and Andrew Thompson), Cryptosporidium parvum, Entamoeba spp. Escherichia coli, Campylobacter jejuni, Campylobacter coli and Salmonella typhimurium was amplified using the species-specific primers for $G$. duodenalis assemblages $\mathrm{A}$ and $\mathrm{E}$, and sequenced. Furthermore, a sub- 
set of the samples was selected for sequencing throughout the study, from both the $G$. duodenalis assemblage A and E-positive samples. The PCR products were purified and sequenced as previously described.

\subsection{Statistical analysis}

The results of the IFA were used to obtain the true calf prevalence, the farm prevalence, the intensity of cyst excretion and the prevalence in each age category. The true calf prevalence was estimated based on the number of negative and positive samples found in the present study, and on the 95\% Probability Intervals (PIs) of the sensitivity (53-97\%) and specificity (91-99\%) of the IFA, as previously described by Geurden et al. (2004). The farm prevalence was calculated as the number of farms with at least one positive calf, compared with the total number of farms in the study. The difference in age between dairy and beef calves, and between $G$. duodenalis assemblage A and E-positive calves, was compared using a two-way Mann-Whitney $U$ test. $P$-values $<0.05$ were considered to indicate a significant difference.

\section{Results}

\subsection{Epidemiological study}

The total number of dairy and beef calves examined for the presence of Giardia cysts, was 499 and 333, respectively. On the dairy farms an average number of five calves was examined, ranging from one to 19 . The mean age of these dairy calves was $26.4 \pm 0.9$ days (mean \pm S.E.M.) ranging from 1 to 70 days, with $59 \%$ younger than 4 weeks. Seventy-four percent of the dairy calves were female and $26 \%$ were male. An average number of seven calves was examined on the beef farms, ranging from one to 17 . The mean age of these beef calves was $32.7 \pm 1.1$ days ranging from 1 to 70 days, with $47 \%$ younger than 4 weeks. Fiftytwo percent of the animals were female and $48 \%$ were male. The mean age of the calves on beef farms was significantly $(P<0.001)$ higher than on dairy farms.

Based on the sensitivity and the specificity estimates of IFA (Geurden et al., 2004) and on the IFA results of the samples collected on 100 dairy and 50 beef farms, the true prevalence of Giardia was estimated (Table 1).

\subsection{Molecular characterisation}

At least one isolate from 32 out of 48 Giardia-positive dairy farms was selected for molecular characterisation.

Table 1

The true prevalence of Giardia (\%) (95\% Probability Intervals in parentheses), for different age classes of dairy and beef calves (less than 4 weeks, 4-10 weeks and overall), together with the farm prevalence

\begin{tabular}{lllll}
\hline Calf type & $<4$ Weeks & $4-10$ Weeks & Overall & Farm prevalence \\
\hline Dairy & $9(2-19)$ & $41(25-61)$ & $22(12-34)$ & 48 \\
Beef & $34(19-53)$ & $55(36-80)$ & $45(30-64)$ & 64 \\
\hline
\end{tabular}

For the beef farms, at least one isolate from 21 of the 32 positive farms was included. From the isolates of the remaining farms, there was either not enough faeces from the positive samples to extract DNA or no amplification product could be obtained from the sample(s). The number of samples included and the results of the molecular characterisation on the $\beta$-giardin gene are presented in Table 2 .

Overall, the livestock-specific G. duodenalis assemblage E $(64 \%)$ was predominantly identified using the $\beta$-giardin gene. In beef calves $(84 \%)$ and in calves with clinical giardiosis $(74 \%)$, the livestock-specific $G$. duodenalis assemblage E was most prevalent, whereas in dairy calves $G$. duodenalis assemblage A $(59 \%)$ was identified in the majority of the samples. Within G. duodenalis assemblage A, the subgenotypes $\mathrm{A} 2$ and $\mathrm{A} 3$, and within $G$. duodenalis assemblage $\mathrm{E}$, the subgenotypes E2 and E3 were identified. In 25 of the 32 dairy farms (78\%) and in 12 of the 21 beef farms (57\%), G. duodenalis assemblage A was identified in at least one isolate. There was no significant difference in the age of the calves infected with $G$. duodenalis assemblage A or E $(P>0.05)$.

The assemblage-specific tpi PCR proved to be a reliable and specific diagnostic tool; only the specific assemblage yielded an amplification product, which proved to be the expected sequence. All control samples were negative (Fig. 1). The specificity of the assemblage-specific PCR was further confirmed throughout the study by the sequencing of assemblage-specific PCR amplification products from dairy, beef and clinical samples. Eighteen of the forty-four samples $(38 \%)$ yielding a positive amplification for the G. duodenalis assemblage A-specific PCR and 33 of the 85 samples (39\%) yielding a positive amplification for the $G$. duodenalis assemblage E-specific PCR, were selected for sequencing. All G. duodenalis assemblage Apositive samples on the assemblage-specific PCR were confirmed as G. duodenalis assemblage A, and all G. duodenalis assemblage E-positive samples were confirmed as G. duodenalis assemblage $\mathrm{E}$. The number of samples included and the results of the assemblage-specific tpi PCR are presented in Table 3. For 19 samples amplified with the $\beta$-giardin gene no result was obtained using the tpi PCR protocol, due to the absence of sufficient DNA material or lack of amplification. In the majority of samples the identification of the $\beta$-giardin gene was confirmed by the identification of the tpi gene, either as a single infection or as mixed G. duodenalis assemblage $\mathrm{A}$ and $\mathrm{E}$ infections. In total, $69 \%$ of the samples identified as G. duodenalis assemblage A and $20 \%$ of the samples identified as $G$. duodenalis assemblage $\mathrm{E}$ by the $\beta$-giardin gene, were identified as mixed infections by the tpi gene.

\section{Discussion}

As in previous studies, the prevalence of Giardia was high in the present study, both in beef and in dairy calves. Due to the frequent identification of Giardia cysts in faecal samples, cattle have long been considered an important source for human infections. Recent studies, however, indi- 
Table 2

Results of the molecular characterisation based on the $\beta$-giardin gene for Giardia in dairy calves, beef calves, clinically affected calves and overall, with the age and age range in days ( $n=$ total number of samples)

\begin{tabular}{lllll}
\hline & $n$ & Assemblage A (\%) & Assemblage E (\%) & Age (range) \\
\hline Dairy & 49 & $29(59)$ & $20(41)$ & $39(11-70)$ \\
Beef & 44 & $7(16)$ & $37(84)$ & $39(6-70)$ \\
Clinical & 27 & $7(26)$ & $20(74)$ & $<352$ \\
Overall & 120 & $43(36)$ & $77(64)$ & \\
\hline
\end{tabular}

Mixed infections were not diagnosed using this PCR protocol. The percentage (\%) of samples identified as the respective Assemblage is presented in parentheses.

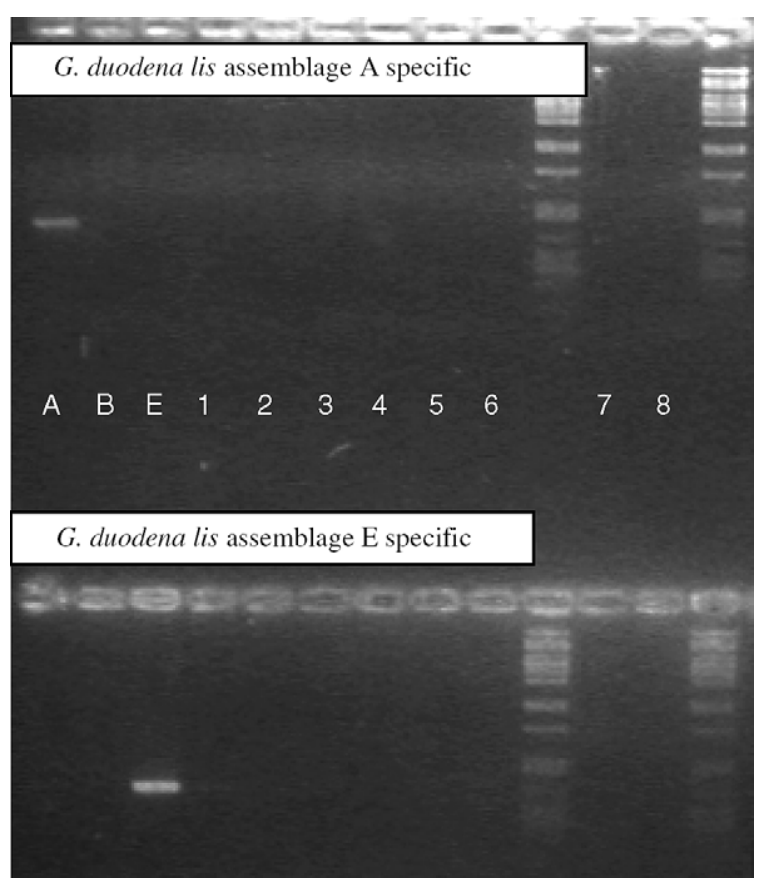

Fig. 1. The assemblage-specific PCR based on the triose-phosphate isomerase gene. In the top lane samples are amplified with the Giardia duodenalis assemblage A-specific primers, and in the bottom lane the samples are amplified with the $G$. duodenalis assemblage E-specific primers. Samples consisted of purified DNA from: (A) G. duodenalis assemblage A; (B) G. duodenalis assemblage $\mathrm{B}$; (E) $G$. duodenalis assemblage E; (1) Entamoeba spp.; (2) Cryptosporidium parvum; (3) Campylobacter jejuni; (4) Campylobacter coli; (5) Salmonella typhimurium; (6) Escherichia coli; (7) negative control of the first reaction; (8) negative control of the nested reaction. Amplification products were visualized on $1.5 \%$ agarose gels with ethidium bromide.

cated that cattle should not be considered as an important source for human giardiosis (Caccio et al., 2005) because most bovine cases of giardiosis are due to infection with
G. duodenalis assemblage E (Hunt et al., 2000; O'Handley et al., 2000; Appelbee et al., 2003; Becher et al., 2004; Trout et al., 2004, 2005, 2006; Langkjaer et al., 2007). Similarly, in the present study, the majority of the Giardia-positive samples were identified as $G$. duodenalis assemblage E based on the $\beta$-giardin gene, although a substantial number of the isolates from dairy calves were $G$. duodenalis assemblage A. In Italy, assemblage A was also found to be most prevalent in calves (Berrilli et al., 2004; Lalle et al., 2005), although a limited number of calf isolates were identified. Both the assemblage A and E subgenotypes found in the present study have previously been identified in calves in other studies (Berrilli et al., 2004; Lalle et al., 2005). Furthermore, the assemblage A2 and A3 subgenotypes found in the calves in the present study have been reported in human patients (Lalle et al., 2005), confirming the relevance of calves as a zoonotic reservoir.

Besides the high prevalence of assemblage A in dairy calves, the assemblage-specific PCR demonstrated the presence of mixed $G$. duodenalis assemblage $\mathrm{A}$ and $\mathrm{E}$ infections not only in dairy calves, but also in beef calves and clinically affected calves. Overall, the majority (69\%) of the $G$. duodenalis assemblage A and $20 \%$ of the G. duodenalis assemblage $\mathrm{E}$ infections found with the $\beta$-giardin gene were identified as mixed G. duodenalis assemblage $\mathrm{A}$ and $\mathrm{E}$ infections using the species-specific PCR. The occurrence of mixed infections illustrates the hazards of evaluating the zoonotic potential of Giardia isolates from animal origin using molecular identification based on a general PCR followed by sequencing. Due to the exponential nature of PCR and the subsequent failure to detect small Giardia sub-populations in the same host (Hopkins et al., 1997), the presence of the zoonotic assemblage A in animal faecal samples might be undetected. Next to epidemiological studies, the novel approach of assemblage-specific amplifi-

Table 3

Results of molecular characterisation based on the triose phosphate isomerase gene for Giardia in dairy calves, beef calves, clinically affected calves and overall, with the age and age range in days ( $n=$ total number of samples)

\begin{tabular}{lrllll}
\hline & $n$ & Assemblage A $(\%)$ & Assemblage E (\%) & Mixed assemblage A/E infection (\%) & Age (range) \\
\hline Dairy & 45 & $11(24)$ & $17(38)$ & $17(38)$ & $39(11-70)$ \\
Beef & 31 & $2(6)$ & $22(71)$ & $7(22)$ & $37(6-64)$ \\
Clinical & 25 & $3(12)$ & $15(60)$ & $31(31)$ & $<352$ \\
Overall & 101 & $16(16)$ & $54(53)$ & \\
\hline
\end{tabular}

Using the triose phosphate isomerase primers the simultaneous detection of Giardia assemblages A and E was possible. The percentage (\%) of samples identified as the respective Assemblage is presented in parentheses. 
cation used here can contribute to clinical studies. In human patients, a difference in clinical outcome or assemblage-specific pathogenicity has been described in patients infected with either assemblage A or B (Homan and Mank, 2001; Read et al., 2002), although mixed infections were not considered in these studies. In the present bovine study, a similar assemblage-specific pathogenicity could not be determined using the assemblage-specific PCR. Furthermore, the identification of mixed and mono-infections with G. duodenalis assemblage A in clinically affected calves seems to contradict the transient nature of an infection with assemblage A in calves (Caccio et al., 2005).

The prevalence of $G$. duodenalis assemblage A was high in the present study, especially in the dairy calves. Moreover, infection with Giardia was widely distributed among farms, as G. duodenalis assemblage A was identified in $78 \%$ of the dairy farms and $57 \%$ of the beef farms. A similarly wide distribution of $G$. duodenalis assemblage A among cattle farms was reported in the United States (Trout et al., 2004, 2005, 2006). The wide distribution of G. duodenalis assemblage A on these farms suggests that G. duodenalis assemblage A is probably more widespread in calves than has been assumed, although not diagnosed by commonly used PCR and sequencing protocols. Furthermore, the province of East Flanders is densely populated and the intensive livestock industry is close to human activity in the area. The high prevalence and wide distribution of assemblage A in calves in the present study might therefore indicate interaction between the human and livestock transmission cycles (Thompson and Monis, 2004). The higher likelihood of anthropozoonotic transmission in an endemic focus has previously been described in dogs, gorillas and cattle (Hopkins et al., 1997; Graczyk et al., 2002; Traub et al., 2004; Uehlinger et al., 2006). However, it is uncertain whether the high prevalence of assemblage $\mathrm{A}$ is due to a frequent and repeated infection of susceptible calves or reflects the ability of assemblage A isolates to persist and spread among calves after introduction into the bovine population.

The occurrence of mixed $G$. duodenalis assemblage A and $\mathrm{E}$ infections suggests that calves, although primarily infected with the livestock-specific $G$. duodenalis assemblage $\mathrm{E}$, are frequently hosts of the zoonotic $G$. duodenalis assemblage A. Calves should therefore be considered as a potential zoonotic reservoir for human infections, although the transmission dynamics need to be further elucidated. Additional studies are needed to determine whether mixed infections occur in other geographical regions.

\section{References}

Altschul, S.F., Madden, T.L., Schaffer, A.A., Zhang, J., Zhang, Z., Miller, W., Lipman, D.J., 1997. Gapped BLAST and PSI-BLAST: a new generation of protein database search programs. Nucleic Acids Res. 25, 3389-3402.

Appelbee, A.J., Frederick, L.M., Heitman, T.L., Olson, M.E., 2003. Prevalence and genotyping of Giardia duodenalis from beef calves in Alberta, Canada. Vet. Parasitol. 112, 289-294.
Becher, K.A., Robertson, I.D., Fraser, D.M., Palmer, D.G., Thompson, R.C.A., 2004. Molecular epidemiology of Giardia and Cryptosporidium infections in dairy calves originating from three sources in Western Australia. Vet. Parasitol. 123, 1-9.

Berrilli, F., Di Cave, D., De Liberato, C., Franco, A., Scaramozzino, P., Orecchia, P., 2004. Genotype characterisation of Giardia duodenalis isolates from domestic and farm animals by SSU-rRNA gene sequencing. Vet. Parasitol. 122, 193-199.

Caccio, S.M., Thompson, R.C.A., McLauchlin, J., Smith, H.V., 2005. Unravelling Cryptosporidium and Giardia epidemiology. Trends Parasitol. 21, 430-437.

Edgar, R.C., 2004. MUSCLE: multiple sequence alignment with high accuracy and high throughput. Nucleic Acids Res. 32, 1792-1797.

Geurden, T., Claerebout, E., Vercruysse, J., Berkvens, D., 2004. Estimation of diagnostic test characteristics and prevalence of Giardia duodenalis in dairy calves in Belgium using a Bayesian approach. Int. J. Parasitol. 34, 1121-1127.

Geurden, T., Vercruysse, J., Claerebout, E., 2006. Field testing of a fenbendazole treatment combined with hygienic and management measures against a natural Giardia infection in calves. Vet. Parasitol. 142, 367-371.

Graczyk, T.K., Bosco-Nizeyi, J., Ssebide, B., Thompson, R.C.A., Read, C., Cranfield, M.R., 2002. Anthropozoonotic Giardia duodenalis genotype (assemblage) A infections in habitats of free-ranging human-habituated gorillas, Uganda. J. Parasitol. 88, 905-909.

Hall, T.A., 1999. BioEdit: a user-friendly biological sequence alignment editor and analysis program for Windows 95/98/NT. Nucleic. Acids Symp. Ser. 41, 95-98.

Homan, W.L., Mank, T.G., 2001. Human giardiosis: genotype linked differences in clinical symptomatology. Int. J. Parasitol. 31, 822-826.

Hopkins, R.M., Meloni, B.P., Groth, D.M., Wetherall, J.D., Reynoldson, J.A., Thompson, R.C.A., 1997. Ribosomal RNA sequencing reveals differences between the genotypes of Giardia isolates recovered from humans and dogs living in the same locality. J. Parasitol. 83, 44-51.

Hunt, C.L., Ionas, G., Brown, T.J., 2000. Prevalence and strain differentiation of Giardia intestinalis in calves in the Manawatu and Waikato regions of North Island, New Zealand. Vet. Parasitol. 91, 7-13.

Itagaki, T., Kinoshita, S., Aoki, M., Itoh, N., Saeki, H., Sato, N., Uetsuki, J., Izumiyama, S., Yagita, K., Endo, T., 2005. Genotyping of Giardia intestinalis from domestic and wild animals in Japan using glutamete dehydrogenase gene sequencing. Vet. Parasitol. 133, 283-287.

Lalle, M., Pozio, E., Capelli, G., Bruschi, F., Crotti, D., Caccio, S.M., 2005. Genetic heterogeneity at the beta-giardin locus among human and animal isolates of Giardia duodenalis and identification of potentially zoonotic subgenotypes. Int. J. Parasitol. 35, 207-213.

Langkjaer, R.B., Vigre, H., Enemark, H.L., Maddox-Hyttel, C., 2007. Molecular and phylogenetic characterization of Cryptosporidium and Giardia from pigs and cattle in Denmark. Parasitology 134, 339-350.

O'Handley, R.M., Olson, M.E., Fraser, D., Adams, P., Thompson, R.C.A., 2000. Prevalence and genotypic characterisation of Giardia in dairy calves from Western Australia and Western Canada. Vet. Parasitol. 90, 193-200.

Olson, M.E., Guselle, N.J., O’Handley, R.M., Swift, M.L., McAllister, T.A., Jelinski, M.D., Morck, D.W., 1997. Giardia and Cryptosporidium in dairy calves in British Columbia. Can. Vet. J. 38, 703-706.

Read, C., Walters, J., Robertson, I.D., Thompson, R.C.A., 2002. Correlation between genotype of Giardia duodenalis and diarrhoea. Int. J. Parasitol. 32, 229-231.

Sulaiman, I.M., Fayer, R., Bern, C., Gilman, R.H., Trout, J.M., Schantz, P.M., Das, P., Lal, A.A., Xiao, L., 2003. Triosephosphate isomerase gene characterization and potential zoonotic transmission of Giardia duodenalis. Emerg. Infect. Dis. 9, 1444-1452.

Thompson, R.C.A., Monis, P.T., 2004. Variation in Giardia: implications for taxonomy and epidemiology. Adv. Parasitol. 58, 69-137.

Traub, R.J., Monis, P.T., Robertson, I., Irwin, P., Mencke, N., Thompson, R.C.A., 2004. Epidemiological and molecular evidence supports the zoonotic transmission of Giardia among humans and dogs living in the same community. Parasitology 128, 253-262. 
Trout, J.M., Santin, M., Greiner, E., Fayer, R., 2004. Prevalence of Giardia duodenalis genotypes in pre-weaned dairy calves. Vet. Parasitol. 124, 179-186.

Trout, J.M., Santin, M., Greiner, E., Fayer, R., 2005. Prevalence and genotypes of Giardia duodenalis in post-weaned dairy calves. Vet. Parasitol. 130, 177-183.

Trout, J.M., Santin, M., Greiner, E.C., Fayer, R., 2006. Prevalence and genotypes of Giardia duodenalis in 1-2 year old dairy cattle. Vet. Parasitol. 140, 217-222.

Uehlinger, F.D., Barkema, H.W., Dixon, B.R., Coklin, T., O'Handley R, M., 2006. Giardia duodenalis and Cryptosporidium spp. in a veterinary college bovine teaching herd. Vet. Parasitol. 142, 213-237.
Van de Peer, Y., De Wachter, R., 1994. TREECON for Windows: a software package for the construction and drawing of evolutionary trees for the Microsoft Windows environment. Comput. Appl. Biosci. $10,569-570$.

van Keulen, H., Macechko, P.T., Wade, S., Schaaf, S., Wallis, P.M., Erlandsen, S.L., 2002. Presence of human Giardia in domestic, farm and wild animals, and environmental samples suggests a zoonotic potential for giardiosis. Vet. Parasitol. 108, 97-107.

Wade, S.E., Mohammed, H.O., Schaaf, S.L., 2000. Prevalence of Giardia sp. Cryptosporidium parvum and Cryptosporidium andersoni (syn. C. muris) in 109 dairy herds in five counties of southeastern New York. Vet. Parasitol. 93, 1-11. 